\title{
A new solar fuels reactor concept based on a liquid metal heat transfer
}

\section{fluid: reactor design and efficiency estimation}

Cansheng Yuan, ${ }^{\mathrm{a}, \mathrm{b}, 1}$ Colby Jarrett, ${ }^{\mathrm{b}}$ William Chueh, ${ }^{\mathrm{d}}$ Yoshiaki Kawajiri ${ }^{\mathrm{a}}$ and Asegun Henry, ${ }^{\mathrm{b}, \mathrm{c}, *}$

${ }^{a}$ School of Chemical \& Biomolecular Engineering, Georgia Institute of Technology, 311 Ferst Drive NW, Atlanta, GA 30332, USA.

${ }^{\mathrm{b}}$ George W. Woodruff School of Mechanical Engineering, Georgia Institute of Technology, 495 Tech Way NW, Atlanta, GA 30332, USA.

c School of Materials Science and Engineering, Georgia Institute of Technology, 771 Ferst Drive, Atlanta, GA 30332, USA .

${ }^{\mathrm{d}}$ Department of Material Science and Engineering, Stanford University, 496 Lomita Mall, Stanford, CA 94035, USA.

KEYWORDS: Thermochemical reactor, liquid metal, partial redox cycles, water splitting, heat recuperation.

ABSTRACT: A new reactor concept for two-step partial redox cycles is presented and evaluated by transient simulation that considers heat and mass transfer along with reaction kinetics. The major difference between the reactor described herein and previous designs is that the conversion from solar to chemical energy is divided into two steps: sunlight-to-thermal energy conversion accomplished with a liquid metal based receiver, and the thermal-to-chemical conversion

* Corresponding author

${ }^{1}$ Present address: BASF Advanced Chemicals Co. Ltd, 333 Jiang Xin Sha Road, Pudong, Shanghai, China, 200137

Author Email addresses: cansheng.yuan@basf.com (C. Yuan), colbyjarrett@gmail.com (C. Jarrett), ykawajiri@chbe.gatech.edu (Y. Kawajiri), ase@gatech.edu (A. Henry)

Abbreviations: OSM (oxygen storage material), HTF (heat transfer fluid), LM (liquid metal), LMHTF (liquid metal heat transfer fluid) 
accomplished with a separately optimized array of reaction chambers. To connect these two conversion steps, liquid metal is used as a high temperature heat transfer fluid that feeds the solar energy captured in the receiver to the reactor. The liquid metal also facilitates efficient heat recuperation $(\sim 80 \%)$ between the reaction chambers. The overall thermal-to-chemical efficiency from the thermal energy in the liquid metal to the chemical energy in the hydrogen fuel is estimated to be $19.8 \%$ when ceria is employed as the reactive oxygen storage material. This estimated efficiency is an order of magnitude higher than previous designs and the reactor concept discussed herein identifies important insights that apply to solar-fuel conversion in general.

\section{Nomenclature}

$\begin{array}{ll}\text { Abbreviations } & \\ \text { HTF } & \text { Heat transfer fluid } \\ \text { LM } & \text { Liquid metal } \\ \text { LMHTF } & \text { Liquid metal heat transfer fluid } \\ \text { OSM } & \text { Oxygen storage material } \\ \text { Symbols } & \\ A_{\text {tec }} & \text { Surface area of the receiver } \\ A_{\text {reactor }} & \text { Surface area associated with the reactor } \\ b_{\text {insulation }} & \text { Thickness of insulation } \\ C_{p} & \text { Heat capacity } \\ d_{32} & \text { OSM pore scale particle size } \\ F & \text { Dimensionless energy factors } \\ h_{\text {insulation }} & \text { Heat transfer coefficient of Insulation } \\ H_{\text {chamber }} & \text { Height of chambers }\end{array}$




\begin{tabular}{|c|c|}
\hline$H H V_{H_{2}}$ & Higher heating value of $\mathrm{H} 2$ \\
\hline$L_{\text {chamber }}$ & Length of chambers \\
\hline$\hat{m}_{\text {OSM }}$ & Effective mass of the OSM \\
\hline$M_{\text {OSM }}$ & Molar mass of the OSM \\
\hline$N_{\text {pipe }}$ & Number of pipes inside of one chamber \\
\hline$n_{\mathrm{H}_{2}}$ & Total hydrogen production in a stage by a chamber \\
\hline$n_{\mathrm{O}_{2}}$ & Total oxygen production in a stage by a chamber \\
\hline$p_{\mathrm{O}_{2}}$ & Partial pressure of oxygen \\
\hline$p_{\text {reactor }}$ & Reactor power \\
\hline$Q$ & Heat input \\
\hline$\dot{q}$ & Heat flux \\
\hline$R_{L M}$ & Inner radius of pipe \\
\hline$R_{\text {Sup }}$ & Outer radius of pipe \\
\hline$R_{\mathrm{OSM}}$ & Outer radius including OMS \\
\hline$R_{\mathrm{fs}}$ & Radius of bulk gas layer \\
\hline$t_{\text {pumping }}$ & Pumping time in reduction step \\
\hline$t_{\text {purging }}$ & Purging time in reduction step \\
\hline$t_{\text {preheat }}$ & Time to preheat the chambers \\
\hline$T$ & Temperature profile \\
\hline$T_{H}$ & Reduction step temperature \\
\hline$T_{H-}$ & Initial Chamber temperature of reduction step \\
\hline$T_{L}$ & Oxidation step temperature \\
\hline$T_{L+}$ & Initial Chamber temperature of oxidation step \\
\hline$u_{L M}$ & Velocity of LM \\
\hline
\end{tabular}




$\begin{array}{ll}u_{\text {gas,inlet }} & \text { Inlet velocity of purging gas } \\ W_{\text {chamber }} & \text { Width of chamber } \\ \Delta t & \text { Cycle time } \\ \Delta \delta & \text { Average change in off-stoichiometry } \\ \delta & \text { Nonstoichiometry } \\ \bar{\delta}_{\text {osm }} & \text { Average nonstoichiometry reached in OSM } \\ \rho & \text { Density } \\ \lambda & \text { Thermal conductivity } \\ \varepsilon & \text { Porosity of OSM } \\ \varepsilon_{S} & \text { Sensible heat recuperation efficiency } \\ \tau & \text { Tortuosity of OSM } \\ \eta_{\text {gas }} & \text { Gas-gas heat exchanger efficiency } \\ \eta_{\text {thermal-chemical }} & \text { Thermal to chemical efficiency } \\ \eta_{\text {solar-hermal }} & \text { Solar to thermal efficiency } \\ & \text { Solar to chemical efficiency }\end{array}$

\section{Introduction}

The idea of using solar energy as high temperature process heat to make fuel has been of interest for more than three decades (Funk and Reinstrom, 1966; Funk, 2001; Nakamura, 1977; Steinfeld, 2005; Steinfeld et al., 1995). This idea has gained increased attention over the last few years as attention has shifted to two-step partial redox cycles (Bader et al., 2013; Chueh and Haile, 2010, 2009; Chueh et al., 2010; Diver et al., 2008; Ermanoski et al., 2013; Furler et al., 2012; Keene et al., 2013; Lapp and Lipiński, 2014; Lapp et al., 2012, 2013; Lipiński et al., 2013; Miller et al., 
2012; Muhich et al., 2013; Siegel et al., 2013; Wei et al., 2013; Zinkevich et al., 2006) based on materials such as ceria, which have demonstrated hundreds of cycles with repeatable performance (Chueh et al., 2010). Two-step partial redox cycles using metal oxides undergo the following two reactions to make a fuel, such as hydrogen from water, which is the primary example discussed herein:

Step 1: Reduction Reaction

$$
\mathrm{M}_{x} \mathrm{O}_{y} \stackrel{T_{\mathrm{H}}}{\longrightarrow} \mathrm{M}_{x} \mathrm{O}_{\mathrm{y}-\delta}+\frac{\delta}{2} \mathrm{O}_{2}(\mathrm{~g})
$$

Step 2: Oxidation Reaction

$$
\delta \mathrm{H}_{2} \mathrm{O}(\mathrm{g})+\mathrm{M}_{x} \mathrm{O}_{\mathrm{y}-\delta} \stackrel{T_{\mathrm{L}}}{\longrightarrow} \mathrm{M}_{x} \mathrm{O}_{y}+\delta \mathrm{H}_{2}(\mathrm{~g})
$$

In these reactions, the solid phase metal oxide serves as an oxygen storage material (OSM), signified by $\mathrm{M}_{x} \mathrm{O}_{y}$. The OSM is heated to a high temperature $T_{H}$ (e.g. $1200-1500^{\circ} \mathrm{C}$ ) and is subjected to a low oxygen pressure ( $P_{\mathrm{O}_{2}}$ ) environment where it endothermically releases oxygen from its lattice. The heat required to break the chemical bonds is supplied by the high temperature solar process heat and the oxygen release is driven by the entropy increase experienced by the $\mathrm{O}_{2}$ molecules upon liberation. After step 1 the OSM is in a reduced state $\mathrm{M}_{x} \mathrm{O}_{y-\delta}$ and is then cooled to a lower temperature $T_{L}$ (e.g. $500-800^{\circ} \mathrm{C}$ ), such that the thermodynamic driving force is reversed and the OSM consumes the oxygen in $\mathrm{H}_{2} \mathrm{O}$ to refill its oxygen vacancies. This second reaction liberates hydrogen thereby producing fuel, while the OSM can be reheated and cycled through these two reaction steps without being consumed. 
From a fundamental perspective, using sunlight as a source of thermal energy provides advantages over photocatalytic approaches, because the entire solar spectrum is utilized, as opposed to only using the high energy portion of the spectrum which is capable of splitting chemical bonds directly. Techno-economic analyses by Stechel et al. have shown that the thermochemical approach to solar fuels can be economically viable, if the solar to fuel efficiency of a system exceeds 20\% (Kim et al., 2012, 2011; Siegel et al., 2013).

The overall efficiency of a solar thermochemical reactor is constrained by a steady state balance between the power density of the fuel output and solar energy input. The energy conversion process from sunlight to chemical energy can be divided into two distinct conversions, namely 1) solar to thermal energy conversion, and 2) thermal to chemical energy conversion. To reach the high temperatures required for the reduction step, concentrated solar radiation is usually trapped in a cavity after entering through a small aperture to minimize reradiation losses. The walls and any structures contained inside the cavity serve as the solar receiver, which absorbs the sunlight and converts it to thermal energy. The thermal energy is then converted to chemical energy in the form of fuel (i.e., via a partial redox cycle) in what we will refer to in the subsequent analysis as the reactor.

Using thermodynamic models that do not necessarily consider kinetics or the details of the reactor, several authors have shown that high solar to chemical efficiency (>20\%) can be achieved (Diver et al., 2008; Ermanoski et al., 2013; Lange et al., 2014; Lapp et al., 2012; Siegel et al., 2013). However, current reactors have efficiencies on the order of 1\% (Chueh et al., 2010; Miller et al., 2012), and improvements have led to peak efficiencies up to 4\% (Furler et al., 2012). This large difference between the efficiencies calculated by models and the values measured in experiments is in part due to the fact that thermodynamic based modelling does not fully capture the transient and non-uniform evolution of temperature and oxygen pressure, which govern the rate of fuel production (Keene et al., 2013). Keene et al. (Keene et al., 2013) showed that when the finite 
limitations on heat and mass transfer as well as chemical kinetics are accounted for in current reactors, the predicted efficiencies are low and the discrepancy with experiments is reconciled. Therefore, a transient model including the heat, mass transfer and reaction kinetics is necessary to correctly predict the efficiency (Keene et al., 2013). In the following we introduce an alternative perspective for designing solar fuels reactors and identify several important issues that can be overcome with a different design concept. We then introduce a new reactor concept that attempts to address these issues and uses a similar modelling procedure as Keene et al. (Keene et al., 2013) to predict its performance. The model shows that significantly higher efficiencies may be possible with a different reactor design.

\section{Power Density Mismatch}

Here, we discuss an important issue that exists in many conventional reactor designs. In conventional designs, the OSM (e.g., ceria) simultaneously serves as receiver and reactor (see Fig. 1a). For the first conversion step from solar to thermal energy, the energy balance on the solar absorbing surfaces can be written as:

$$
\dot{q}_{\text {sun }} A_{\text {rec }}=\dot{q}_{\text {heat }} A_{\text {rec }}+\dot{q}_{\text {loss }} A_{\text {rec }}
$$

where $\dot{q}_{\text {sun }}, \dot{q}_{\text {heat }}$, and $\dot{q}_{\text {loss }}$ are fluxes $\left(\mathrm{kW} / \mathrm{m}^{2}\right)$ of the incident solar radiation, thermal energy captured by the receiver, and the parasitic heat loss to the environment, respectively, and $A_{\text {rec }}$ is the surface area of the receiver. Here, it is important to emphasize that $A_{\text {rec }}$ is the macroscopic surface area and not the microscopic surface area that can be obtained from microscopic porosity in the receiver walls. Using these definitions, one can define a solar-to-thermal efficiency $\eta_{\text {solar-thermal }}$ as: 


$$
\eta_{\text {solar-thermal }}=\frac{\dot{q}_{\text {heat }} A_{\text {rec }}}{\dot{q}_{\text {sun }} A_{\text {rec }}}=\frac{\dot{q}_{\text {heat }}}{\dot{q}_{\text {sun }}}
$$

Similarly, the thermal to chemical efficiency $\eta_{\text {thermal-chemical }}$ can be described as

$$
\eta_{\text {thermal-chemical }}=\frac{\dot{q}_{\text {OSM }} A_{\text {reactor }}}{\dot{q}_{\text {heat }} A_{\text {rec }}}
$$

where $\dot{q}_{\text {OSM }}$ is the cycle averaged flux of chemical energy (fuel) production, and $A_{\text {reactor }}$ is the projected surface area associated with the reactor. Here, it is again important to emphasize that $A_{\text {reactor }}$ is the macroscopic surface area of the reactor and not the microscopic surface area that can be obtained from microscopic porosity in the OSM itself, which is needed to facilitate the chemical reactions. With this definition, the chemical energy flux $\dot{q}_{\text {OSM }}$ can be expressed using the properties of the OSM as follows:

$$
\dot{q}_{\mathrm{OSM}}=\frac{n_{\mathrm{H}_{2}} H H V_{\mathrm{H}_{2}}}{A_{\text {reactor }} \Delta t}=\frac{\hat{m}_{\mathrm{OSM}} \Delta \delta}{M_{\text {OSM }}} \frac{1}{\Delta t} \frac{H H V_{\mathrm{H}_{2}}}{A_{\text {reactor }}}
$$

In Eq. (6), $n_{\mathrm{H}_{2}}$ is the amount of hydrogen produced per cycle, $H H V_{\mathrm{H}_{2}}$ is the higher heating value of hydrogen, $\Delta t$ is the cycle time. In the second expression in Eq. (6), $M_{\text {OSM }}$ is molar mass of the OSM; $\hat{m}_{O S M}$ is the effective mass of the OSM that produces fuel during the partial redox cycle, where $\Delta \delta$ represents the average change in off-stoichiometry and the product $\hat{m}_{O S M} \Delta \delta$ simply yields the total amount of oxygen produced during each cycle. Note that $\hat{m}_{O S M}$ is not necessarily the amount of OSM mass in the reactor, as it is possible that not all of the OSM mass is fully cycled through $\Delta \delta$. In this sense, $\hat{m}_{\text {OSM }}$ could be limited by the way heat is delivered 
(i.e., the penetration depth of incoming sunlight), or other transport limitations such as gas transport. By combining Eqs. (4) and (5), the solar-to-chemical efficiency can be expressed as:

$$
\eta_{\text {solar-chemical }}=\eta_{\text {solar-thermal }} \eta_{\text {thermal-chemical }}=\frac{\dot{q}_{\text {oSM }} A_{\text {reactor }}}{\dot{q}_{\text {sun }} A_{\text {rec }}}
$$

As is conceptually illustrated in Fig. 1a, many reactor designs employ direct irradiation of the OSM with sunlight (Chueh et al., 2010; Diver et al., 2008; Furler et al., 2012; Miller et al., 2012), which is intuitive and straightforward. This approach ensures that the endothermic heat required for step 1 is supplied at a sufficiently high rate. However, this approach has an important consequence, which is that it fundamentally links the surface area available for light absorption to the surface area available for chemical reactions ( $A_{\text {reactor }}=A_{\text {rec }}$ in Eq. (7)), and thus the overall efficiency is given by $\eta_{\text {solar-chemical }}=\dot{q}_{O S M} / \dot{q}_{\text {sun }}$. To obtain a high efficiency, this constraint requires $\dot{q}_{\text {osm }}$ to be nearly equal to $\dot{q}_{\text {sun }}$. These two fluxes, however, differ by more than an order of magnitude in previous studies (Chueh et al., 2010; Furler et al., 2012). Based on experimental work of Chueh et al. (Chueh et al., 2010), the average power associated with the fuel power flux production $\dot{q}_{\text {OsM }}$ is only $1.8 \mathrm{~kW} / \mathrm{m}^{2}$, which is obtained from the second equality in Eq. (6) using the values in Table 1 . On the other hand, $\dot{q}_{\text {sun }}=p_{\text {sun }} / A_{\text {rec }}$ is as high as $230 \mathrm{~kW} / \mathrm{m}^{2}$, which is computed from the solar power $p_{\text {sun }}$ of $1.9 \mathrm{~kW}$ for the receiver surface area of $A_{\text {rec }}=0.0082 \mathrm{~m}^{2}$. Therefore, even though a material such as ceria can be cycled many times with minimal degradation, the average power associated with the fuel power flux production $\dot{q}_{\text {osm }}$ is only on the order of $2 \mathrm{~kW} / \mathrm{m}^{2}$, while $\dot{q}_{\text {sun }}$ is on the order of $100 \mathrm{~kW} / \mathrm{m}^{2}$. In concept, it may be possible to achieve much larger values for $\dot{q}_{\text {osm }}$, for example if full instead of partial redox cycles are used. However, for partial redox cycles, which have garnered interest because of the large number of 
repeated cycles that have been demonstrated with minimal degradation, the values for $\dot{q}_{\text {osm }}$ are not likely to increase by an order of magnitude or more, because they fundamentally require that one only cycle a fraction of the constituent oxygen in the lattice.

Table 1. Parameters used to compute $\dot{q}_{\text {osm }}$ with experimental data

\begin{tabular}{ll}
\hline Value & Source \\
\hline$\hat{m}_{\text {OSM }}=325 \mathrm{~g}$ & Total mass of ceria used (Chueh et al., 2010) (assumed to be equal to the effective \\
& mass) \\
$\Delta \delta=0.066$ & Nonstoichiometry $\Delta \delta$ for equilibrium at $T_{H}=1500^{\circ} \mathrm{C} \quad$ and $p_{\mathrm{O}_{2}}=10^{-5}$ atm \\
& (Chueh and Haile, 2010) \\
$\Delta t=40 \mathrm{~min}$ & Cycle time, obtained from Fig. 3 in Chueh et al. (Chueh et al., 2010) \\
$d_{\text {inner }}=25.7 \mathrm{~mm}_{\text {reactor }}=0.0082 \mathrm{~m}^{2}$ & Inner diameter of cylindrical OSM (Chueh et al., 2010) \\
& \\
$h_{\text {OSM }}=102 \mathrm{~mm}$ & Height of cylindrical OSM (Chueh et al., 2010)
\end{tabular}

The difference of two orders of magnitude between the power input $\dot{q}_{\text {sun }}$ and output $\dot{q}_{\text {osm }}$ is an important power density mismatch, and offers a very simple explanation for why the efficiency of conventional reactors are on the order of $1-2 \%$. This is because the rate at which heat is fed into reactors greatly exceeds the rate at which fuel can be extracted. As a result, a steady state energy balance requires that the remaining heat be lost. Keene et al.(Keene et al., 2013) have shown that reradiation losses dominate as the cavity continues to increase in temperature until a stagnation occurs where the majority of the heat is reradiated from the cavity. 
Although there are several potential strategies to increase the efficiency $\eta_{\text {solar-chemical }}$ for conventional designs, substantial technical challenges must be overcome. One intuitive solution to improve the efficiency is to increase $\dot{q}_{\text {OSM }}$ either by improvement of the reactor design or material. Referring to Eq. (6), for the reactor design improvement, $\dot{q}_{\text {osm }}$ can be increased by increasing the effective mass of ceria in the reactor $\hat{m}_{O S M}$. However, for conventional designs, increasing the effective mass is fundamentally limited by how far the sunlight can penetrate into the OSM. This is determined by the solar flux distribution, the OSM optical properties and how fast oxygen can be removed from the reactor. One would not expect to gain an order of magnitude increase in such quantities and therefore we believe this pathway may not yield order of magnitude improvements.

For material improvements, increasing $\Delta \delta$ can also increase $\dot{q}_{\text {OSM }}$. However, for materials such as ceria, $\Delta \delta$ is limited to values on the order of $0.01-0.1$ for the temperatures and oxygen pressures accessible in previous experiments (Chueh et al., 2010; Furler et al., 2012; Miller et al., 2012). Thus, it is also unlikely that order of magnitude improvements can be realized by increasing $\Delta \delta$. Finally, the cycle time $\Delta t$ may be reduced to compensate for the two order of magnitude difference between $\dot{q}_{\text {sun }}$ and $\dot{q}_{\text {OSM }}$. However, order of magnitude shorter cycle times would require extremely fast heating and cooling rates, which may not be possible because of material limitations associated with thermal shock or system level heat transfer limitations. Therefore, it is difficult to envision a two order of magnitude increase in $\dot{q}_{\text {osm }}$ with current materials and reactor designs.

Another approach for increasing the efficiency $\eta_{\text {solar-chemical }}$ could be to reduce $\dot{q}_{\text {sun }}$. This can be realized by reducing the ratio of the aperture area to the OSM area. While this approach may be 
viable, it will likely require careful engineering to minimize heat leakage while maintaining the high temperatures required for the reactions. It may also be challenging to achieve fast cycle times, since the heat input at a given location will be much lower, leading to slower heating rates. To the best of our knowledge, such an approach has never been attempted. In practice, the opposite has been observed; experiments done by Furler et al. found that increasing $\dot{q}_{\text {sun }}$, increased efficiency (Furler et al., 2012). The reason efficiency increased in their case was due to a higher value of $\dot{q}_{\text {OSM }}$, as the higher $\dot{q}_{\text {sun }}$ resulted in a higher reduction temperature which increased $\Delta \delta$, and reduced the cycle time $\Delta t$ more than the increased reradiation loss. This approach, however, ultimately corresponds to an increase in $\Delta \delta$, and thus is expected to have similar limitations as was previously mentioned, specifically for partial redox materials that can be repeatedly cycled without degradation.

\section{A New Reactor Concept}

Since $\dot{q}_{\text {osm }}$ and $\dot{q}_{\text {sun }}$ are naturally disparate for current materials, here we consider a different approach, which decouples the two conversion processes such that $A_{\text {reactor }}$ need not be equal to $A_{\text {rec }}$. Such a reactor concept would allow $A_{\text {reactor }}$ to be significantly greater than $A_{\text {rec }}$. The tradeoff, however, is likely to be an increase in capital cost for the now larger reactor, but it could potentially be viable if $\eta_{\text {solar-chemical }}$ is drastically improved and the fuel generated is of sufficiently high value.

One approach to decoupling the receiver and reactor area is to use a moving/flowing media for sunlight absorption. For the region of the system that is illuminated by the concentrated sunlight, one cannot limit its radiative view factor back to the environment at the location of sunlight exposure, due to the requirement of detailed balance. One can decrease the aperture size to 
generally decrease the view factor back to the environment, but this increases the required concentration, which is both fundamentally limited and practically limited by issues such as the finite size of the sun (e.g., it is not a point source) and tracking accuracy. For the region of the system where concentrated sunlight absorption occurs, the input flux is usually so large that the time scale required to heat a medium to the target reduction temperature is short, by comparison to the time scale required to generate the fuel. Thus, it is advantageous to decouple these two time scales, by using a moving/flowing medium that passes through the region of high sunlight concentration and high view factor to the environment quickly. Then, once the medium has reached the target peak reduction temperature $T_{H}$, the medium can be moved to another region of the system where its view factor to the environment is effectively zero (e.g., a location completely surrounded by thermal insulation), preventing it from losing the heat that was captured. Once in this enclosed portion of the system, the medium can be utilized to perform the thermal-tochemical conversion in a way that may take advantage of a much larger surface area, which is likely to be needed for sufficiently fast reaction kinetics. As a result, the usage of a moving/flowing medium fundamentally allows one to separate the area of sunlight exposure for solar-to-thermal conversion from the larger area needed for thermal-to-chemical conversion.

This approach has been pursued previously, for example, by Koepf et al.(Koepf et al., 2012) and Ermanoski et al.. In their work, the OSM moves/flows as particles (Ermanoski et al., 2013), which are only temporarily exposed to the incident light, whereby they are heated to the target temperature. Once heated, the particles flow into a portion of the system that has effectively zero view factor to the environment (e.g., fully enclosed containment) instead of remaining fixed in the region where sunlight is concentrated. This then allows the time required for heating to be completely decoupled from the time required for chemical reactions as these approaches can operate as batch processes. In these reactor concepts, the total surface area available for chemical reactions $\left(A_{\text {reactor }}\right)$ is proportional to the surface area of all the particles being cycled and is 
decoupled from the surface area available for sunlight absorption ( $A_{\text {rec }}$ ), which is proportional to the receiver aperture size.

Recognizing that decoupling the receiver and reactor surface is critical, here we examine another approach that realizes the same advantages as Koepf et al.(Koepf et al., 2012) and Ermanoski et $a l$. , but also facilitates improved recuperation. The new reactor concept introduced herein uses two separate devices, namely an optimized solar-thermal receiver and a separately optimized thermal-chemical reactor, that are connected through a high temperature heat transfer fluid (HTF). This concept is schematically illustrated in Fig. 1(b). In this way, the exposed surface area and materials needed for the solar-thermal conversion are decoupled from the thermal-chemical conversion. In such a design, we can increase $A_{\text {reactor }}$ while keeping $A_{\text {rec }}$ fixed, which allows us to dramatically increase solar to chemical efficiency. This decoupling can be accomplished with a solar receiver, which uses a liquid HTF to absorb sunlight at a high temperature. The HTF is then pumped to a thermochemical reactor to deliver the heat to a stationary OSM and produce fuel.

The reactor concept shown in Fig. 1(b) hinges on the usage of a high temperature HTF that is both liquid and chemically stable in the temperature range of interest or $\left[T_{L}, T_{H}\right.$ ]. Molten salts (Hasuike et al., 2006; Lata et al., 2008; Xu and Wiesner, 2012), glasses (Deubener et al., 2009) and metals (Sharafat and Ghoniem, 2000; Subasic, 1998) are among the only classes of fluids that remain in the liquid phase at temperatures of approximately $1,000^{\circ} \mathrm{C}$ or above. Among these choices, liquid metals have a number of advantages that could prove useful for the application of thermochemical reactors as follows (Wetzel et al., 2014): (1) metals such as Sn have low melting points $\left(232^{\circ} \mathrm{C}\right)$, high boiling points $\left(2602^{\circ} \mathrm{C}\right)$ and low viscosities, similar to water at temperatures slightly above their melting point. This is an advantage over molten glasses, which can become highly viscous and difficult to pump at lower temperatures; (2) metals such as Sn are thermodynamically stable in contact with a variety of ceramics, which can be used as 
containment materials with no corrosion. This is an advantage not necessarily shared by molten salts or glasses, which often contain a larger number of elements facilitating formation of unwanted corrosion products; (3) liquid metals have thermal conductivities that are approximately two orders of magnitude higher than non-electrically conductive liquids. This leads to much higher convective heat transfer coefficients and thereby reduces the pumping power required for high rates of heat transfer.

Given these potential advantages we have designed a new type of solar fuels reactor that uses liquid metal (LM) as a HTF and can therefore overcome not only the issues associated with the power density mismatch, but can also facilitate highly efficient solid phase heat exchange, which has been identified as major source of inefficiency (Lapp et al., 2012; Nakamura, 1977; Steinfeld et al., 1999). Since it is chemically compatible with liquid Sn at all temperatures and is easily machinable, it is currently envisioned that the system will be primarily constructed out of graphite. To correctly predict the reactor efficiency, a transient model is built based on our design. As is shown later, our model suggests that our new design can exhibit thermal-to-chemical efficiencies $\eta_{\text {thermal-chemical }}$ of approximately $20 \%$. It is important to note that, to our knowledge, no previous reactor design that has been modeled in detail has shown that such high efficiencies can be realized (Keene et al., 2013; Lapp and Lipiński, 2014).

In the following, we discuss the reactor model and performance, assuming a solar receiver that uses a liquid metal heat transfer fluid (LMHTF) can facilitate the solar-thermal conversion at high efficiency ( $\eta_{\text {solar-thermal }} \sim 0.8-0.9$ ) and supplies the reactor with LMHTF at the peak reactor temperature $T_{H}$. The ensuing analysis is not contingent on this assumption, as the following analysis is devoted specifically to $\eta_{\text {thermal-chemical }}$. Thus, the assumption of high efficiency solar to thermal conversion simply serves as a basis by which the independent examination of $\eta_{\text {thermal-chemical }}$ is justified, since it is expected to be the limiting efficiency for the entire system 
(see Eq. (7)). Nonetheless, it is useful to note that the idea of a $80-90 \%$ efficient solar receiver appears feasible based on modeling results presented elsewhere (DeAngelis and Henry, 2014), which rely on a graphite receiver held in an inert environment. Such a receiver can reach such high efficiencies, when the concentration entering the cavity is $\geq 5000 \mathrm{~kW} / \mathrm{m}^{2}$, whereby the flux on the receiver absorbing surfaces is on the order of $100 \mathrm{~kW} / \mathrm{m}^{2}$. For a material such as graphite, which has a thermal conductivity $>20 \mathrm{~W} / \mathrm{mK}$ at high temperature $\left(1500^{\circ} \mathrm{C}\right)$ (Uher, 1991), a receiver made of tubes or walls with thicknesses on the order of $1 \mathrm{~cm}$ has a conductive thermal resistance of $<5 \times 10^{-4} \mathrm{~m}^{2} \mathrm{~K} \mathrm{~W}^{-1}$, and can therefore transfer the heat to the $\mathrm{LM}$ with a temperature drop across the wall on the order of a few tens of degrees $\mathrm{K}(<50 \mathrm{~K})$. This then minimizes the temperature difference between the sunlight absorbing cavity surfaces that reradiate back to the environment and the LM exit temperature. This then yields high efficiencies commensurate with the receiver efficiencies obtained in current concentrated solar power (CSP) plants. Since the issue of the receiver efficiency is to be examined elsewhere, the ensuing discussion is devoted to the working principles of the reactor and its novel heat recovery strategy. The modeling results

provide new insights and guidance on new directions that can be pursued for even further improvements in reactor efficiency.

\section{Efficiency Considerations}

As discussed in the preceding section, in this study we examine an approach based on a LMHTF. In this approach a receiver, which can be located separately from the reactor, heats a LMHTF such as $\mathrm{Sn}(\mathrm{l})$. After being heated in the receiver, the LMHTF is pumped to the reactor to supply the thermal energy to the thermochemical cycle for fuel generation. In the ensuing discussion we have focused specifically on hydrogen production, via water splitting, however, other fuels or fuel precursors are also possible, such as syngas (Bader et al., 2013; Chueh and Haile, 2010, 2009; Chueh et al., 2010; Diver et al., 2008; Ermanoski et al., 2013; Funk and Reinstrom, 1966; Funk, 2001; Furler et al., 2012; Keene et al., 2013; Kim et al., 2012, 2011; Lapp and Lipiński, 2014; 
Lapp et al., 2013, 2012; Lipiński et al., 2013; Loutzenhiser et al., 2010; Miller et al., 2012; Muhich et al., 2013; Nakamura, 1977; Siegel et al., 2013; Steinfeld, 2005; Steinfeld et al., 1999, 1995; Wei et al., 2013; Xu and Wiesner, 2012; Zinkevich et al., 2006). The high thermal conductivity of the LMHTF allows for extremely power dense heat transfer (i.e. 1-10 MW/m²) and is one of the principle reasons our models indicate that the receiver can operate with high efficiency $\left(\eta_{\text {solar-thermal }} \sim 80-90 \%\right)$ at $1350^{\circ} \mathrm{C}$ (DeAngelis and Henry, 2014).

In our current design, a general form of the thermal efficiency $\eta_{\text {thermal-chemical }}$ is given by:

$$
\eta_{\text {thermal-chemical }}=\frac{n_{\mathrm{H}_{2}} H H V_{\mathrm{H}_{2}}}{Q_{\text {Total }}}=\frac{n_{\mathrm{H}_{2}} H H V_{\mathrm{H}_{2}}}{Q_{\text {Reheat }}+Q_{\text {Loss }}+W_{\text {Pump }}+Q_{\text {Purge }}+Q_{R X N}}
$$

In Eq. (8), $Q_{\text {Total }}$ is the total energy input per cycle; $Q_{\text {Rereat }}$ accounts for the thermal energy required to heat the OSM and reactor from $T_{L}$ to $T_{H} ; Q_{\text {Loss }}$ accounts for the heat leakage from the entire reactor system; $Q_{R X N}$ represents the endothermic energy required to liberate oxygen during the reduction step; $W_{\text {Pump }}$ accounts for the mechanical work input required to lower the $\mathrm{O}_{2}$ partial pressure if a vacuum pump is used; $Q_{\text {Purge }}$ accounts for the energy required to preheat the purge gas to $T_{L}$ to $T_{H}$. Detailed formulations for these energy inputs are discussed in section 6 . To better understand how each term in the denominator of (Eq. (8)) limits the efficiency, we define dimensionless energy factors $F_{i}$, where each energy requirement is normalized with respect to the total amount of fuel produced:

$$
F_{i}=Q_{i} /\left(n_{\mathrm{H}_{2}} H H V_{\mathrm{H}_{2}}\right)
$$

where $i=$ Reheat, Loss, Work, and Purge. In this way, the numerator of Eq. (8) becomes unity, and it allows us to more easily identify the efficiency bottlenecks: 


$$
\eta_{\text {thermal-chemical }}=\frac{1}{F_{\text {Reheat }}+F_{\text {Loss }}+F_{\text {Work }}+F_{\text {Purge }}+F_{\text {RXN }}}
$$

Previous analyses have established that $F_{\text {Reheat }}$ is one of the most important limiting factors in the efficiency (Bader et al., 2013; Chueh et al., 2010; Diver et al., 2008; Ermanoski et al., 2013; Furler et al., 2012; Keene et al., 2013; Lapp and Lipiński, 2014; Lapp et al., 2013, 2012; Lipiński et al., 2013; Loutzenhiser et al., 2010; Miller et al., 2012; Siegel et al., 2013; Steinfeld, 2005; Steinfeld et al., 1999; Wei et al., 2013), aside from reradiation losses. This is because, for partial redox cycles, all of the atoms in the lattice, not only the oxygen, must be heated from $T_{L}$ to $T_{H}$. Therefore, by comparison, the sensible heating requirement $F_{\text {Reheat }}$ can greatly outweigh $F_{R X N}$. There have been successful approaches to addressing this issue, such as the radiative recuperation strategy of the CR5 (Diver et al., 2008; Miller et al., 2012), and the CR5 simulations demonstrate recuperative efficiencies $>$ 50\% (Lapp and Lipiński, 2014; Lapp et al., 2013). Since our system attempts to solve the reradiation problem using a LMHTF based receiver, we focused our reactor design on solving the recuperation problem, since it can be the largest loss in the reactor.

The second major issue addressed by our design is the desire to control the gas environment separately for the two reaction steps. As will be discussed later, it is desirable to operate the reduction reaction at reduced total pressure and the oxidation reaction at higher pressures. This, however, is not an option in some reactor designs where there is no hermetic seal between the OSM undergoing the reduction step and OSM undergoing the oxidation step (Diver et al., 2008; Miller et al., 2012).

With these issues in mind, in our design we use an array of sealed reaction chambers that are interconnected with a piping network which allows the LMHTF to transfer heat between the chambers (see Fig. 2(a) and (d)). In our system all reaction chambers matriculate through the two reaction steps at different times and therefore have different temperatures at any given instant. In 
this reactor concept, the two reaction steps (Eqs. (1) and (2)), as well as the heat recuperation, occur cyclically and semi-continuously in separate reaction chambers. Each individual reaction chamber consists of an array of pipes (see Fig. 2(b)), contained inside a hermetically sealed outer housing. Each pipe serves as a containment material for the LMHTF flowing through its inner bore (see Fig. 2(b) and (c)). Each pipe also serves as a support for the OSM as the OSM is coated around its outer diameter. At present, we envision using graphite as the piping material that supports the OSM, particularly because it is machinable, chemically compatible with $\mathrm{Sn}(\mathrm{l})$ at any temperature of interest, has high thermal conductivity at high temperature and can withstand the repeated thermal cycling between $T_{L}$ and $T_{H}$ (Sato et al., 1975).

A thin fully dense diffusion barrier, such as a $\mathrm{ZrO}_{2}$ based layer, is also needed as a coating on the pipes to block chemical interactions between the OSM and the pipe, as well as chemical interactions between the gas environment and the pipe. Current development efforts suggest that a $\mathrm{C}-\mathrm{ZrC}-\mathrm{ZrO}_{2}-\mathrm{OSM}$ layering approach may offer sufficient protection. Thus far, considering all of the materials requirements, a materials solution seems feasible, but additional engineering will likely be needed to increase lifetime and reliability. Such issues are beyond the scope of the current investigation, but attempting to solve those challenges must first be motivated by the potential to achieve much higher efficiency, as is assessed by our model and ensuing discussion.

\section{Reactor Design and Operation}

During the endothermic reduction step, high temperature LMHTF from the solar receiver is pumped through the parallel array of pipes inside one of the reaction chambers. The sensible heat of the LMHTF is first transferred via convection to the pipe's inner wall, then it is conducted through the pipe wall to the OSM coated on the outside. The heat then conducts through the OSM to its surface where oxygen atoms are liberated, which results in a local cooling effect. While the high temperature LMHTF from the receiver flows through the inside of the pipes coated with the 
OSM, separate gas ports on the hermetically sealed container control the gas environment experienced by the OSM on the outside of each pipe, as shown in Fig. 3.

During the reduction step, a vacuum pump is first used to lower the total pressure to approximately $10^{-2}$ atm by opening Valve 2 with the vacuum pump working at the exit of reaction chamber, which reduces the total pressure in the reactor to a desired level. Once the target pressure is reached, Valves 1 and 3 are opened, and Valve 2 is shut off to enable high velocity low pressure steam to enter the reaction chamber, which acts as a purge gas and further reduces the $P_{\mathrm{O}_{2}}$. It is important to appreciate that these gas valves need not operate at elevated temperatures and therefore one can make use of elastomer based sealing approaches applicable at room temperature. During the reduction step, the total pressure is kept to the value at the end of the pumping step, so that the steam is supplied at low pressure. The reduced pressure decreases the density of the gas, thereby reducing the total amount of heat needed to preheat the gas to $T_{H}$. Using a low-pressure sweep gas has been experimentally evaluated by Miller et al. (Miller et al., 2012). Here, the sweep gas energy penalty scales with the ratio of the purging gas flow rate to the $\mathrm{O}_{2}$ flow rate, which is approximately $P_{\text {sweep }} / P_{\mathrm{O}_{2}}$ (Ermanoski et al., 2013). Therefore if low $\mathrm{O}_{2}$ pressures are desired, low total pressures are needed to minimize this ratio.

The idea of using steam as a purge gas is unconventional, but is motivated by its easier separation from $\mathrm{O}_{2}$ in the product stream by simply condensing out $\mathrm{H}_{2} \mathrm{O}$ in the liquid phase. During the high temperature reduction reaction, one concern is that steam may simply reoxidize the OSM, counteracting the intended reduction reaction. Steam that enters the chamber partially dissociates to generate an effective oxygen partial pressure associated with the equilibrium between water and hydrogen in the gas phase. At the temperatures of interest, the resulting partial pressure of oxygen is $\sim 10^{-5}$ atm. As will be shown later, this oxygen pressure is significantly lower than the lowest oxygen pressure achieved in our reaction chambers, due to constraints associated with the 
cycle time and limiting the losses from preheating the purge gas. Thus it is expected that steam can be used as a purge gas without causing any unwanted reoxidation during step 1 .

During the gas recuperation from the product stream, the heat released upon condensation can be recycled to vaporize more steam forming a circulation loop as shown in Fig. 4, which shows the concept of using a heat exchanger, condenser, and evaporator in a circulation loop. Here, we envision using a heat exchanger to preheat the purge gas with high temperature outlet stream from reaction chambers. The condenser separates the product $\mathrm{H}_{2}$ and $\mathrm{O}_{2}$ from liquid water, which is supplied to the evaporator to regenerate purge gas. This avoids the need for mixed gas separations to recover a pure oxygen stream.

In the reactor system shown in Fig. 2(a), while one reaction chamber undergoes the reduction step, the chamber on the opposite side of the circle undergoes the water splitting oxidation step. In this reaction chamber, higher pressure (i.e. $1 \mathrm{~atm}$ ) steam enters through the gas ports as the reactant for the water splitting reaction. A mixture of $\mathrm{H}_{2} \mathrm{O}+\mathrm{H}_{2}$ exits the reactor and its sensible heat can be recuperated through a heat exchanger, which is similar to the system used for the high temperature purging steam discussed above for reduction. Again, by simply using pure water as the input, it can be separated from the $\mathrm{H}_{2}$ fuel that is produced by condensing out the water and using the heat of condensation to vaporize more reactant. Thus, the primary reason for using steam is to enable separations with minimal energy requirements since the heat of condensation can be recuperated.

While two of the reaction chambers undergo the reduction and oxidation steps, the other chambers in the circle recuperate the heat through the pipes at intermediate temperatures between $T_{L}$ and $T_{H}$. Each reaction chamber has a set of pipes that carry LMHTF to and from every other reactor in the circle. This piping network is used exclusively for heat recuperation and consists of $N(N-1)$ pipes, where $N$ is the total number of reaction chambers in the circle. This 
expression accounts for the fact that circulation of LMHTF between reaction chambers requires both a send and receive channel.

Fig. 5 shows two stages (Stages 1 and 2) in the cyclic operation of the proposed reactor concept. Each stage consists of three steps-pumping, purging, and preheating-as described below. In the pumping step in Stage 1, Chamber 1 undergoes the reduction step at the initial temperature of $T_{H-}$, which is below the desired temperature $T_{H}$, while Chamber 5 undergoes the oxidation step at $T_{L_{+}}$. All other chambers are at intermediate temperatures between $T_{H_{-}}$and $T_{L_{+}}$. During the pumping step, Chamber 1 is heated to the desired temperature $T_{H}$ by the LMHTF from the solar receiver. Simultaneously the reduction reaction (Equation (1)) occurs while the oxygen pressure is reduced by the vacuum pump. On the opposite side, Chamber 5 is cooled from $T_{L+}$ to $T_{L}$, and then undergoes the oxidation step (Equation (2)). Simultaneously, all remaining chambers transfer thermal energy through the LMHTF to recuperate heat between themselves. After the pressure in Chamber 1 is reduced to about $10^{-2}$ atm, purging is performed (purging step) to further reduce $P_{\mathrm{O}_{2}}$ for reduction reaction, while the heat recuperation and oxidation reaction still continue. After purging is completed, the preheating step begins, where the supply of LMHTF from the solar receiver stops, and Chamber 1 at the highest temperature $T_{H}$ transfers the heat to Chamber 2. Simultaneously, Chamber 5 at the lowest temperature $T_{L}$ exchange heat with Chamber 6. The rest of chambers transfer thermal energy between themselves via the LMHTF to recuperate the heat as well. After completing the above three steps, the next stage, Stage 2 begins, where Chamber 2 undergoes reduction step and Chamber 6 performs the oxidation step. The remaining stages follow a similar sequence. With this heat recuperation scheme, the sensible heat stored in the contents of each reaction chamber can be recuperated without mechanically moving 
the OSM. Instead, the LMHTF is moved, which reduces the number of solid moving parts to those contained in the pumps and valves associated with the LMHTF circulation loops.

With this approach the theoretical maximum recuperation efficiency $\mathcal{E}_{S-M A X}$ simply depends on the number of chambers $N$. If one assumes constant heat capacity for all materials and no heat loss, the maximum recuperation efficiency is $\varepsilon_{S-M A X}=1-1 / N$ (see Supporting Information). From this equation, it can be seen that with this new recuperation scheme based on the usage of a LMHTF, one can achieve high recuperation efficiencies (> 80\% with eight reactors). The idea is easily generalized to any number of reactors, but a larger number of reactors translates to a larger capital cost, but higher recuperation efficiency.

We summarize the advantages of the proposed concept below. One of the principal benefits of our approach is that the surface area/power density associated with the reactor can be separately optimized from that of the receiver. If the energy flux of the OSM $\dot{q}_{\text {OSM }}$ is low, then the area for chemical reactions $A_{\text {reactor }}$ can be increased by simply increasing the number and/or density of pipes coated with the OSM within each reaction chamber, thereby increasing the efficiency $\eta_{\text {thermal-chemical }}$ according to Eq. (5). This illustrates how separating the receiver and reactor can improve efficiency, which is the primary benefit of the proposed reactor concept. The second major benefit of our new reactor concept is that the usage of a HTF facilitates efficient heat recovery of the solid phase and minimizes the number of moving parts. The third major benefit of our reactor concept arises from the use of an array of reaction chambers, which allows one to separately control the gas environments during each reaction step. 


\section{Reactor Model}

The reactor model consists of transient coupled mass and energy balance equations for all phases in the reaction chamber (the bulk gas, OSM, OSM support, and the LMHTF) as well as the reaction kinetics. The model was implemented in gPROMS to identify limiting parameters to improve the efficiency (Process Systems Enterprise Ltd., 2004).

In the model, the thermochemical efficiency $\eta_{\text {thermal-chemical }}$ is given by,

$$
\eta_{\text {thermal-chemical }}=\frac{n_{\mathrm{H}_{2}} H H V_{\mathrm{H}_{2}}}{Q_{\text {Total }}}=\frac{n_{\mathrm{H}_{2}} H H V_{\mathrm{H}_{2}}}{Q_{\text {Reheat }}+Q_{\text {Loss }}+W_{\text {Pump }}+Q_{\text {Purge }}+Q_{R X N}}
$$

where $W_{\text {Pump }}$ is the energy required by the pump, and $Q_{\text {Reheat }}, Q_{\text {Loss }}, Q_{\text {Purge }}$, and $Q_{R X N}$ are heat input for the sensible heat for OSM, heat input to compensate the heat loss to the environment, heat to increase the temperature of the purge gas, and the heat of reaction, respectively. Here, $Q_{\text {Reheat }}, Q_{\text {Loss }}$, and $Q_{R X N}$ is given by,

$$
\begin{gathered}
Q_{\text {Reheat }}=\left(1-\varepsilon_{S}\right) \int_{T_{L}}^{T_{H}}\left(m_{\text {OSM }} C_{P}^{\text {OSM }}+m_{\text {Sup }} C_{P}^{\text {Sup }}\right) d T \\
Q_{\text {Loss }}=\dot{Q}_{\text {Loss }} \Delta t \\
Q_{R X N}=n_{O_{2}} \Delta H_{O_{2}}
\end{gathered}
$$

The energy input for the pump $W_{\text {Pump }}$ is given by:

$$
W_{\text {Pump }}=\int \frac{\dot{n}_{\text {gas }}(P(t))}{\eta_{\text {Pump }}(P(t))} R T_{\text {pump }} \ln \left(\frac{P(t)}{P^{a t m}}\right) d t
$$

where $P^{a t m}$ is atmospheric pressure, $\dot{n}_{\text {gas }}$ is the molar flow rate of gas which is a function of reaction chamber pressure $P(t), \quad \eta_{\text {Pump }}$ is the pump efficiency which is also function of $P(t)$. Finally, $Q_{\text {Purge }}$ is given by:

$$
Q_{\text {Purge }}=\left(1-\varepsilon_{G}\right) \cdot \int_{T_{\infty}}^{T} C_{P}{ }^{g a s} n_{\text {gas }} d T
$$


where $T_{\infty}$ is the ambient temperature, $T$ is the temperature of the reactor (which is either $T_{L}$ or $\left.T_{H}\right), n_{\text {gas }}$ is the total amount of purge gas used, and $\varepsilon_{G}$ is the efficiency of the gas heat exchanger. More detailed discussions on the assumptions and sensitivity analysis are given in Yuan et al (submitted).

The transient model consists of mass and energy balance equations for all layers (gas, OSM, OSM support, and the LMHTF shown in Fig. 6) of a single pipe in the reaction chamber as well as the reaction kinetics. Additional discussion about the model as well as the approach to efficiency optimization are presented elsewhere Yuan et al. (submitted).

The heat transfer between the OSM and LMHTF layers is given by the following equation, where the convective heat transfer rate is characterized by the heat transfer coefficient $h_{L M}$,

$$
\rho_{\mathrm{LM}} C_{P}^{L M} \frac{\partial T_{L M}}{\partial t}+\rho_{L M} C_{P}^{L M} u_{L M} \frac{\partial T_{L M}}{\partial z}-\lambda_{L M} \frac{\partial^{2} T_{L M}}{\partial z^{2}}=A_{L M} h_{L M}\left(T_{\text {Sup }}\left(R_{\text {Sup }}, z, t\right)-T_{L M}(z, t)\right)(17)
$$

where $A_{L M}$ is the surface area to volume ratio for the pipe. The energy balance within the OSM support layer is then given by the following equation,

$$
\rho_{\text {Sup }} C_{p} \text { Sup } \frac{\partial T_{\text {Sup }}}{\partial t}-\lambda_{\text {Sup }}\left(\frac{\partial^{2} T_{\text {Sup }}}{\partial R^{2}}+\frac{1}{R} \frac{\partial T_{\text {Sup }}}{\partial R}+\frac{\partial^{2} T_{\text {Sup }}}{\partial \mathrm{z}^{2}}\right)=0
$$

Similarly, the energy balance within the OSM layer is given by,

$$
\rho_{O S M} C_{P}^{O S M} \frac{\partial T_{O S M}}{\partial t}-\lambda_{O S M}\left(\frac{\partial^{2} T_{O S M}}{\partial R^{2}}+\frac{1}{R} \frac{\partial T_{O S M}}{\partial R}+\frac{\partial^{2} T_{O S M}}{\partial z^{2}}\right)=r_{i} \Delta H_{i}
$$

where $r_{i} \Delta H_{i}$ is the source term due to the heat of reaction. The energy balance in the bulk gas layer is given by the following equation,

$$
\rho_{\text {gas }} C_{P}^{g a s} \frac{\partial T_{\text {gas }}}{\partial t}+\rho_{\text {gas }} C_{P}^{\text {gas }} \frac{\partial\left(u_{\text {gas }} T_{\text {gas }}\right)}{\partial z}-\lambda_{\text {gas }} \frac{\partial^{2} T_{\text {gas }}}{\partial z^{2}}=A_{O S M} h_{\text {gas }}\left(T_{\text {OSM }}\left(R_{O S M}, z, t\right)-T_{\text {gas }}(z, t)\right)
$$

where $A_{O S M}$ is surface area to volume ratio for the OSM layer.

In the reaction chamber, the $\mathrm{O}_{2}$ or $\mathrm{H}_{2}$ molecules produced in the OSM layer diffuse from the surface of an OSM particle to the top of the OSM layer. The rate of the diffusion is represented by the effective diffusion coefficient $D_{\text {eff }}$, while the rate of mass transfer for the molecules that travel across the boundary layer between the bulk gas layer and OSM is described by mass transfer coefficient $k_{\text {gas }}$. For the OSM layer, the mass balance equation considers the diffusion through the OSM layer and surface reaction rate, as follows,

$$
\varepsilon \frac{\partial c_{i, O S M}}{\partial t}-\varepsilon D_{e f f}\left(\frac{\partial^{2} c_{i, O S M}}{\partial R^{2}}+\frac{1}{R} \frac{\partial c_{i, O S M}}{\partial R}+\frac{\partial^{2} c_{i, O S M}}{\partial z^{2}}\right)=-r_{i}
$$


where $\varepsilon$ is the porosity of OSM. For the bulk gas layer, the mass balance equation can be written as follows,

$$
\frac{\partial c_{i, g a s}}{\partial t}+u_{g a s} \frac{\partial c_{i, g a s}}{\partial \mathrm{z}}+c_{i, g a s} \frac{\partial u_{\text {gas }}}{\partial \mathrm{z}}+k_{\text {gas }} A_{\text {OSM }} \varepsilon\left(c_{i, g a s}(z, t)-c_{i, \mathrm{OSM}}\left(R_{O S M}, z, t\right)\right)=0
$$

The gas velocity $u_{\text {gas }}$ is obtained from the overall mass balance equation for all species,

$$
\frac{\partial c_{\text {Total }}}{\partial t}+u_{\text {gas }} \frac{\partial c_{\text {Total }}}{\partial z}+c_{\text {Total }} \frac{\partial u_{\text {gas }}}{\partial z}+\sum k_{\text {gas }} A_{\text {OSM }} \varepsilon\left(c_{i, g a s}(z, t)-c_{i, \text { OSM }}\left(R_{\text {OSM }}, z, t\right)\right)=0
$$

Using Kroger Vink notation, the reaction rate for oxygen production is then be given by (Keene et al., 2013),

$$
r_{\mathrm{O}_{2}}(R, z, t)=\frac{k_{\mathrm{O}_{2}}^{+}}{2}\left\{\frac{(1-2 \delta)^{2}(1-0.5 \delta)}{2 K_{K V}}-\left(\frac{p_{\mathrm{O}_{2}}(R, z, t)}{p_{r e f}}\right)^{\frac{1}{2}} \delta^{3}\right\}
$$

Here, the reaction rate constant $k_{\mathrm{O}_{2}}^{+}$is given by $k_{\mathrm{O}_{2}}^{+}=k_{\mathrm{O}_{2}} A_{R}=k_{\mathrm{O}_{2}} \frac{6(1-\varepsilon)}{d_{32}}$, where $A_{R}$ is the specific surface area, and $d_{32}$ is the particle diameter of the OSM material and the equilibrium constant $K_{K V}$ is given as,

$$
K_{K V}=\exp \left(-\frac{\Delta \bar{h}_{K V}-T \Delta \bar{s}_{K V}}{R T}\right)
$$

where $\Delta \bar{h}_{K V}$ and $\Delta \bar{s}_{K V}$ are enthalpy and entropy of the reduction reaction, which can be obtained from the experimental data provided by Panlener and Blumenthal (Panlener and Blumenthal, 1975). The nonstoichiometry $\delta$ can then be computed from the following (Keene et al., 2013),

$$
\frac{\partial}{\partial t}\left\{\frac{\rho_{\mathrm{MO}_{2}}}{M_{\mathrm{MO}_{2}}}(1-\varepsilon) \delta(R, z, t)\right\}=k_{\mathrm{O}_{2}}^{+}\left\{\frac{(1-2 \delta)^{2}(1-0.5 \delta)}{2 K_{K V}}-\left(\frac{p_{\mathrm{O}_{2}}}{p_{\text {ref }}}\right)^{\frac{1}{2}} \delta(R, z, t)^{3}\right\}
$$

Here, the reaction rate constant $k_{\mathrm{O}_{2}}$ is obtained by fitting Equation (14) to experimental data (Chueh and Haile, 2010), since the precise kinetics are not known. This approach is equivalent to simply assuming the OSM chemistry and microstructure are the same as that which was measured by Chueh and Haile (Chueh and Haile, 2010). Testing the sensitivity of the overall system performance revealed that this choice is not critical because the reoxidation step is not necessarily rate limiting for such structures. As a result, the usage of a more accurate kinetic model is not expected to alter the conclusions of the study herein, and the kinetics used in this model are physically realizable.

For the oxidation reaction, the hydrogen production rate is given by: 


$$
r_{H_{2}}(R, z, t)=\frac{k_{H_{2}}^{+}}{2} \delta^{m}\left(\frac{p_{\mathrm{H}_{2} \mathrm{O}}}{p_{\text {ref }}}\right)^{0.54}
$$

where $k_{H_{2}}^{+}$is given by $k_{H_{2}}^{+}=k_{H_{2}} A_{R}=k_{H_{2}} \frac{6(1-\varepsilon)}{d_{32}}$. The change in nonstoichiometry is then given by,

$$
\frac{\partial}{\partial t}\left\{\frac{\rho_{\mathrm{MO}_{2}}}{M_{\mathrm{MO}_{2}}}(1-\varepsilon) \delta(R, z, t)\right\}=k_{\mathrm{H}_{2}}^{+} \delta^{m}\left(\frac{p_{\mathrm{H}_{2} \mathrm{O}}}{p_{\text {ref }}}\right)^{0.54}
$$

where the two parameters, the reaction rate constant $k_{H_{2}}$ and reaction order $m$, are obtained by fitting Equation (17) to experimental data (Chueh and Haile, 2009).

In our model, we assume that there are many parallel pipes $\left(N_{\text {pipes }}\right)$, such that the output of an entire reaction chamber (shown in Figure 2(b)) is well approximated by simply modeling the behavior a single pipe and multiplying by $\mathrm{N}_{\text {pipes }}$. Under this approximation, simulating one model allows us to estimate the performance of the entire chamber, where all extensive variables such as flow rates and energy expenditures in the chamber are equally divided amongst the array. The same assumption has been made for adsorption processes (Rezaei et al., 2014), which can be justified by appropriately designing the distributor at the gas inlet and collector at the gas outlet (Luo and Tondeur, 2005).

We finally note that any reaction at an intermediate temperature between $T_{H}$ and $T_{L}$ is ignored in this model, thus making our estimate of $\eta_{\text {thermal-chemical }}$ conservative. If the reaction is allowed to continue at the intermediate temperatures in between, it will simply result in a larger fuel output and efficiency, since no additional energy input is required. Nonetheless, further improvement of the estimates provided herein would require the reaction rates to be characterized over a wide range of temperatures, which is presently beyond the scope of this study. 


\section{Heat recuperation and efficiency estimation}

For recuperation of the sensible heat in the OSM and reaction chambers, pairs of chambers exchange the LM, and the same operation is repeated in a cyclical manner (see Fig. 5). In this study, we assume $T_{H}=1500^{\circ} \mathrm{C}$ and $T_{L}=800^{\circ} \mathrm{C}$ based on the experimental data obtained by Chueh and Haile (Chueh and Haile, 2010, 2009). To determine the time necessary for the heat recuperation, the start-up of the entire recuperation scheme is simulated using an energy balance, and the transient dynamics are simulated. For this simulation, the initial temperature of all chambers is set to $1450 \mathrm{~K}$, to see how fast the cyclic steady state can be reached. Figure 7 shows the average temperature of each of the eight reaction chambers as a function of time. In Fig. 7, the switching time between stages is 12 minutes, which includes 8 minutes of reaction time and 4 min of preheating time. It can be seen that within 12 minutes all chambers reach thermal equilibrium, and thus 12 minutes is sufficient to complete each heat recuperation step. As a result 12 min intervals for each stage of the cycle results in each reactor reaching an intermediate quasiequilibrium state, such that additional time is not likely to drastically change the results.

With this simulation, we can also determine the efficiency of the sensible heat recuperation. Blue and green solid lines with circle markers in Fig. 7 represent the initial temperature of the reduction and oxidation reaction, which reach the temperatures of $T_{H-}=1410^{\circ} \mathrm{C}$ and $T_{L+}=877^{\circ} \mathrm{C}$, respectively in one cycle. Therefore, the sensible heat that needs to be supplied from an external source is only needed to heat the highest temperature chamber from $T_{H-}$ to $T_{H}$, while heat required to reach $T_{H-}$ is recuperated. The recuperation efficiency is then defined as,

$$
\varepsilon_{s}=\frac{Q_{T_{L} \rightarrow T_{H-}}}{Q_{T_{L} \rightarrow T_{H-}}+Q_{T_{H-} \rightarrow T_{H}}+Q_{\text {loss }}^{N}+Q_{\text {loss }}^{\text {pipes }}}
$$


where $Q_{T_{L} \rightarrow T_{H-}}$ is the sensible heat recuperated, $Q_{T_{H-} \rightarrow T_{H}}$ is the sensible heat supplied from the solar receiver for the reduction step, and $Q_{\text {loss }}^{N}$ and $Q_{\text {loss }}^{\text {pips }}$ are the additional heat loads that must be supplied to the reactors and pipes, respectively, to keep the entire system at elevated temperature. The sum of these two values, $Q_{\text {loss }}^{N}$ and $Q_{\text {loss }}^{\text {pipes }}$ can be estimated via a simple thermal resistance circuit,

$$
Q_{\text {loss }}^{N}+Q_{\text {loss }}^{\text {pipes }}=\left(A_{\text {chamber }}^{N}+A_{\text {pipes }}\right)\left(T_{\text {Reactor }}-T_{\infty}\right) \Delta t /\left(\frac{b_{\text {insulation }}}{\lambda_{\text {insulation }}}+\frac{1}{h_{\text {insulation }}}\right)
$$

where $A$ and $T_{\text {Reactor }}$ are corresponding surface area and temperature responsible for heat losses, $b_{\text {insulation }}$ is the thickness of insulation layer that covers the entire reaction chamber and all pipes, $\lambda_{\text {insulation }}$ and $h_{\text {insulation }}$ are the thermal conductivity and convective heat transfer coefficient for insulation layer, respectively. Since the temperatures of the chambers are evenly distributed between $T_{L}$ and $T_{H}$, we take the reactor temperature as the average temperature $T_{\text {Reactor }}=\left(T_{L}+T_{H}\right) / 2$. The surface area of pipes can be estimated from diagram of Fig. 8 and the recuperation efficiency is calculated to be 0.81 , which is $93 \%$ of the maximum value $\varepsilon_{S-M A X}=1-1 / N=0.875$ (See Supplementary Information). The main difference between the two efficiencies is the inclusion of heat losses in $\varepsilon_{S}$.

We simulated the reduction and reoxidation reactions using the mathematical model introduced in section 6 and the model/design parameters are summarized in Table 2. The two reactions occur simultaneously in reaction chambers on opposing sides of the circle shown in Fig. 5. It should be noted that the results here are associated with a reactor designed to produce a time average of 1 $\mathrm{kW}$ fuel output (25 $\mathrm{g} / \mathrm{hr}$ of $\mathrm{H}_{2}$ ) and the reactor performance parameters are shown in Table 3. The 
variables $n_{\mathrm{O}_{2}}$ and $n_{\mathrm{H}_{2}}$ represent the products generated in one $12 \mathrm{~min}$ stage, and the average nonstoichiometry $\bar{\delta}_{\text {OSM }}$ is,

$$
\bar{\delta}_{\mathrm{OSM}}=\frac{\int_{0}^{L_{\text {chamber }}} \int_{R_{\text {Sup }}}^{R_{\text {OSM }}} \delta\left(R, t_{\text {preheat }}+t_{\text {pumping }}+t_{\text {purging }}, z\right) 2 \pi R d R d z}{\pi\left(R_{\text {OSM }}^{2}-R_{\text {Sup }}^{2}\right) L_{\text {chamber }}}
$$

Table 2. Reactor chamber design parameters and operating parameters used for this simulation

\begin{tabular}{|c|c|c|c|c|c|}
\hline Parameters & Value & Unit & Parameters & Value & Unit \\
\hline \multicolumn{3}{|c|}{ Reactor design parameters } & \multicolumn{3}{|c|}{ Oxidation reaction } \\
\hline$W_{\text {chamber }}$ & 0.2 & $\mathrm{~m}$ & $T_{L}$ & 800 & ${ }^{\circ} \mathrm{C}$ \\
\hline$H_{\text {chamber }}$ & 0.2 & $\mathrm{~m}$ & $T_{L+}$ & 877 & ${ }^{\circ} \mathrm{C}$ \\
\hline$L_{\text {chamber }}$ & 0.6 & $\mathrm{~m}$ & $u_{\text {gas, inlet }}$ & 0.08 & $\mathrm{~m} \mathrm{~s}^{-1}$ \\
\hline$\eta_{\text {gas }}$ & 0.7 & & \multicolumn{3}{|c|}{ OSM properties } \\
\hline$N_{\text {pipe }}$ & 45 & & $\rho_{\text {OSM }}$ & 7215 & $\mathrm{~kg} \mathrm{~m}^{-3}$ \\
\hline$R_{L M}$ & 0.005 & $\mathrm{~m}$ & $C_{p}^{O S M}$ & 358 & $\mathrm{~J} \mathrm{~kg}^{-1} \mathrm{~K}^{-1}$ \\
\hline$R_{\text {Sup }}$ & 0.01 & $\mathrm{~m}$ & $\lambda_{\mathrm{OSM}}$ & 0.2 & $\mathrm{~W} \mathrm{~m}^{-1} \mathrm{~K}^{-1}$ \\
\hline$R_{\text {OSM }}$ & 0.013 & $\mathrm{~m}$ & $\varepsilon$ & 0.6 & \\
\hline$R_{f s}$ & 0.0168 & $\mathrm{~m}$ & $d_{32}$ & 10.0 & $\mu m$ \\
\hline$u_{L M}$ & 0.03 & $\mathrm{~m} \mathrm{~s}^{-1}$ & $\tau$ & 1.2 & \\
\hline \multicolumn{3}{|c|}{ Reduction reaction } & \multicolumn{3}{|c|}{ Insulation properties } \\
\hline$T_{H}$ & 1500 & ${ }^{\circ} \mathrm{C}$ & $b_{\text {insulation }}$ & 0.3 & $\mathrm{~m}$ \\
\hline$u_{\text {gas,inlet }}$ & 5.0 & $\mathrm{~m} \mathrm{~s}^{-1}$ & $\lambda_{\text {insulation }}$ & 0.05 & $\mathrm{~W} \mathrm{~m}^{-1} \mathrm{~K}^{-1}$ \\
\hline$T_{H-}$ & 1410 & ${ }^{\circ} \mathrm{C}$ & $h_{\text {insulation }}$ & 5.0 & $\mathrm{~W} \mathrm{~m}^{-1} \mathrm{~K}^{-1}$ \\
\hline$t_{\text {pumping }}$ & 0.5 & $\min$ & & & \\
\hline
\end{tabular}




$\begin{array}{lll}t_{\text {purging }} & 7.5 & \min \\ t_{\text {preheat }} & 4.0 & \min \end{array}$

Table 3. Reactor performance for eight chamber reactor system

\begin{tabular}{lcc}
\hline Output & Value & Unit \\
\hline$\eta_{\text {thermal-chemical }}$ & $19.8 \%$ & \\
$n_{\mathrm{O}_{2}}$ & 1.20 & $\mathrm{~mol} / \mathrm{stage}$ \\
$n_{\mathrm{H}_{2}}$ & 2.40 & $\mathrm{~mol} / \mathrm{stage}$ \\
$\bar{\delta}_{\text {OSM }}$ & & \\
& 0.0245 & \\
$p_{\text {reactor }}$ & & \\
& 0.95 & $\mathrm{~kW}$ \\
\hline
\end{tabular}

The efficiency of converting thermal energy in the LMHTF to the chemical energy stored in hydrogen can be computed from Eq. (8). Figure 9(a) shows each energy expenditure for the cycle, all of which affect the efficiency. Fig. 9(a) shows that the heat contributed to the endothermic heat of reaction $F_{R X N}$ is about 32\% of the total heat requirement, which is the second largest. The fact that $F_{R X N}$ is a large fraction of the energy expended indicates that our reactor design is efficient. It should also be noted that the energy required to preheat the steam is only $3.0 \%$ for both the reduction and oxidation reaction. This is because the density of the purging steam is low, due to the low pressure, and $70 \%$ of the supplied heat is recuperated with the heat exchanger system, which is slightly more conservative than the recuperator efficiencies assumed by Lapp et al. (Lapp et al., 2012) and Ermanoski et al. (Ermanoski et al., 2013). Since the vacuum pump was only used for 30 seconds while its efficiency is above 1\% (e.g., at higher pressures of 1.0-0.01 
atm), the energy consumption for the pump is also small. The pumping power needed to flow the LMHTF is on the order of $0.01 \mathrm{~kW}$, which is an order of magnitude smaller than power required to reheat the purge gas and is therefore negligible.

We note that although nearly $80 \%$ of the sensible heat $\left(F_{R}\right)$ is recuperated in the proposed system, the unrecovered heat which is $20 \%$ of the total sensible heat still consumes approximately $37 \%$ of the total heat input ( $F_{\text {Reheat }}$ in Fig. 9(a)). One way to alleviate this issue is to increase the thermal mass ratio of the OSM to the pipes and chamber walls, such that a larger fraction of the recuperated heat is used to heat the OSM and not the supporting materials.

Another significant energy loss is heat leakage to the environment. This loss is contained in $F_{\text {Loss }}$, and can be decreased by increasing the volume-to-surface area ratio for the reactor system as shown in Fig. 9(b). Here, the ratio of the volume to surface area ${ }^{\phi}$ for a rectangular enclosure for the entire reactor is,

$$
\phi=\frac{V_{\text {chamber }}}{A_{\text {insulation }}}=\frac{W_{\text {chamber }} L_{\text {Chamber }} H_{\text {Chamber }}}{2\left(W_{\text {chamber }} L_{\text {Chamber }}+L_{\text {Chamber }} H_{\text {Chamber }}+W_{\text {Chamber }} H_{\text {Chamber }}\right)}
$$

where $\mathrm{W}, \mathrm{H}$ and $\mathrm{L}$ represent the width, height and length, respectively. Thus, the volume to surface area ratio $\phi$ is determined by the aspect ratio of the reaction chamber as well as its overall size for a rectangular geometry. For this geometry, one can determine, by inspection that the maximum value of $\phi$, for a fixed volume, occurs for a cubic reaction chamber ( $L_{\text {chamber }}=W_{\text {chamber }}=H_{\text {chamber }}$ ), where $\phi=L_{\text {Chamber }} / 6$. Since $F_{\text {Loss }}$ is proportional to the surface area of the reactor, while all other heat inputs are proportional to the volume of the reactor, increasing $\phi$ leads to a decrease in $F_{\text {Loss }}$ and at sufficiently large scales this loss can be almost 
completely suppressed with an insulation material that covers the entire chamber of a fixed thickness $b_{\text {insulation }}$.

Although the model of our new reactor concept predicts high efficiencies, we note there are still a number of remaining technical challenges and uncertainties, most of which are the subject of ongoing work towards testing a functional prototype. The discussion herein only focuses on the efficiency of the reactor and not the solar receiver, which is examined elsewhere (DeAngelis and Henry, 2014). Furthermore, there are many technical challenges that remain before the proposed reactor can be demonstrated. For example, as previously mentioned, interactions between the gas environment and piping material, as well as the OSM itself and the pipe material must be prevented since it is envisioned to use graphite for the LM piping network and overall structure. This necessitates development of a fully dense diffusion barrier that must be adhere to the graphite or possibly other substrate material, through repeated thermal cycles. In addition, all materials that contact the LMHTF must be stable at the high temperatures required and will experience repeated and fast thermal cycling. Lastly, the design modeled herein would require high temperature LM pumps and valves. Such components would likely need to be made entirely from refractories such as graphite or ceramics, with graphite packing materials used for sealing. Such components are feasible and are currently being tested as part of an ongoing effort. Nonetheless, the high efficiencies predicted by the model suggest that the new reactor concept presented herein is worthwhile to pursue, as the materials and engineering challenges are not insurmountable.

\section{Conclusions}

The power density mismatch in current thermochemical reactor designs, stimulated the development of an innovative reactor system for two step water splitting via partial redox cycle, which has been evaluated herein. LMHTF is adopted as the heat transfer medium and it serves as 
the heat source for the reactor. One important improvement compared to previous systems in the literature is the recuperation of sensible heat by circulating the LMHTF between the chambers. The piping network between reactors was also simulated and the results show that this strategy can recuperate $80 \%$ of the heat stored in the reactors and OSM, or more if additional reaction chambers are used. The efficiency in converting the LMHTF sensible heat to the hydrogen fuel is estimated to be $19.8 \%$. This new design has significant room for improvement as well as a variety of technical challenges, which must be addressed in future work.

\section{Acknowledgement}

This project was supported by DOE ARPA-E, grant number DE-AR0000339.

\section{References}

Bader, R., Venstrom, L., Davidson, J., Lipiński, W., 2013. Thermodynamic analysis of isothermal redox cycling of ceria for solar fuel production. Energy \& Fuels 27, 5533-5544.

Chueh, W.C., Falter, C., Abbott, M., Scipio, D., Furler, P., Haile, S.M., Steinfeld, A., 2010. Highflux solar-driven thermochemical dissociation of $\mathrm{CO} 2$ and $\mathrm{H} 2 \mathrm{O}$ using nonstoichiometric ceria. Science 330, 1797-801. doi:10.1126/science.1197834

Chueh, W.C., Haile, S.M., 2010. A thermochemical study of ceria: exploiting an old material for new modes of energy conversion and CO2 mitigation. Philos. Trans. A. Math. Phys. Eng. Sci. 368, 3269-94. doi:10.1098/rsta.2010.0114

Chueh, W.C., Haile, S.M., 2009. Ceria as a thermochemical reaction medium for selectively generating syngas or methane from $\mathrm{H} 2 \mathrm{O}$ and CO2. ChemSusChem 2, 735-9. doi:10.1002/cssc.200900138

DeAngelis, A., Henry, A., 2014. Sensitivity Analysis of a High Temperature Liquid Metal Based Solar Receiver, in: Proceedings of the 11th AIAA/ASME Joint Thermophysics and Heat Transfer Conference.

Deubener, J., Helsch, G., Moiseev, a., Bornhöft, H., 2009. Glasses for solar energy conversion systems. J. Eur. Ceram. Soc. 29, 1203-1210. doi:10.1016/j.jeurceramsoc.2008.08.009

Diver, R.B., Miller, J.E., Allendorf, M.D., Siegel, N.P., Hogan, R.E., 2008. Solar Thermochemical Water-Splitting Ferrite-Cycle Heat Engines. J. Sol. Energy Eng. 130, 041001. doi:10.1115/1.2969781 
Ermanoski, I., Siegel, N.P., Stechel, E.B., 2013. A New Reactor Concept for Efficient SolarThermochemical Fuel Production. J. Sol. Energy Eng. 135, 031002. doi:10.1115/1.4023356

Funk, J.E., 2001. Thermochemical hydrogen production: past and present. Int. J. Hydrogen Energy 26, 185-190. doi:10.1016/S0360-3199(00)00062-8

Funk, J.E., Reinstrom, R.M., 1966. Energy requirements in the production of hydrogen from water. Ind. Eng. Chem. Process Des. Dev. 5, 336-342.

Furler, P., Scheffe, J., Gorbar, M., Moes, L., 2012. Solar thermochemical CO2 splitting utilizing a reticulated porous ceria redox system. Energy \& Fuels 26, 7051-7059.

Hasuike, H., Yoshizawa, Y., Suzuki, A., Tamaura, Y., 2006. Study on design of molten salt solar receivers for beam-down solar concentrator. Sol. Energy 80, 1255-1262. doi:10.1016/j.solener.2006.03.002

Keene, D.J., Davidson, J.H., Lipiński, W., 2013. A Model of Transient Heat and Mass Transfer in a Heterogeneous Medium of Ceria Undergoing Nonstoichiometric Reduction. J. Heat Transfer 135, 052701. doi:10.1115/1.4023494

Kim, J., Henao, C.A., Johnson, T.A., Dedrick, D.E., Miller, J.E., Stechel, E.B., Maravelias, C.T., 2011. Methanol production from CO2 using solar-thermal energy: process development and techno-economic analysis. Energy Environ. Sci. 4, 3122. doi:10.1039/c1ee01311d

Kim, J., Johnson, T.A., Miller, J.E., Stechel, E.B., Maravelias, C.T., 2012. Fuel production from CO2 using solar-thermal energy: system level analysis. Energy Environ. Sci. 5, 8417. doi:10.1039/c2ee21798h

Koepf, E., Advani, S.G., Steinfeld, A., Prasad, A.K., 2012. A novel beam-down, gravity-fed, solar thermochemical receiver/reactor for direct solid particle decomposition: Design, modeling, and experimentation. Int. J. Hydrogen Energy 37, 16871-16887. doi:10.1016/j.ijhydene.2012.08.086

Lange, M., Roeb, M., Sattler, C., Pitz-Paal, R., 2014. T-S diagram efficiency analysis of two-step thermochemical cycles for solar water splitting under various process conditions. Energy 67, 298-308. doi:10.1016/j.energy.2014.01.112

Lapp, J., Davidson, J.H., Lipiński, W., 2013. Heat Transfer Analysis of a Solid-Solid Heat Recuperation System for Solar-Driven Nonstoichiometric Redox Cycles. J. Sol. Energy Eng. 135, 031004. doi:10.1115/1.4023357

Lapp, J., Davidson, J.H., Lipiński, W., 2012. Efficiency of two-step solar thermochemical nonstoichiometric redox cycles with heat recovery. Energy 37, 591-600. doi:10.1016/j.energy.2011.10.045

Lapp, J., Lipiński, W., 2014. Transient Three-Dimensional Heat Transfer Model of a Solar Thermochemical Reactor for H 2 O and CO 2 Splitting Via Nonstoichiometric Ceria Redox Cycling. J. Sol. Energy Eng. 136, 031006. doi:10.1115/1.4026465 
Lata, J.M., Rodríguez, M., de Lara, M.A., 2008. High Flux Central Receivers of Molten Salts for the New Generation of Commercial Stand-Alone Solar Power Plants. J. Sol. Energy Eng. 130, 021002. doi:10.1115/1.2884576

Lipiński, W., Davidson, J.H., Haussener, S., Klausner, J.F., Mehdizadeh, a. M., Petrasch, J., Steinfeld, a., Venstrom, L., 2013. Review of Heat Transfer Research for Solar Thermochemical Applications. J. Therm. Sci. Eng. Appl. 5, 021005. doi:10.1115/1.4024088

Loutzenhiser, P.G., Meier, A., Steinfeld, A., 2010. Review of the Two-Step H2O/CO2-Splitting Solar Thermochemical Cycle Based on Zn/ZnO Redox Reactions. Materials (Basel). 3, 4922-4938. doi:10.3390/ma3114922

Luo, L., Tondeur, D., 2005. Optimal distribution of viscous dissipation in a multi-scale branched fluid distributor. Int. J. Therm. Sci. 44, 1131-1141. doi:10.1016/j.ijthermalsci.2005.08.012

Miller, J.E., Allendorf, M.A., Ambrosini, A., Coker, E.N., Diver, R.B., Ermanoski, I., Evans, L.R., Hogan, R.E., Mcdaniel, A.H., 2012. Development and assessment of solar-thermalactivated fuel production: phase 1 summary, Sandia Report.

Muhich, C.L., Evanko, B.W., Weston, K.C., Lichty, P., Liang, X., Martinek, J., Musgrave, C.B., Weimer, A.W., 2013. Efficient generation of $\mathrm{H} 2$ by splitting water with an isothermal redox cycle. Science 341, 540-2. doi:10.1126/science.1239454

Nakamura, T., 1977. Hydrogen production from water utilizing solar heat at high temperatures. Sol. Energy 19, 467-475.

Process Systems Enterprise Ltd., 2004. gPROMS Introductory User Guide. gPROMS Introd. User Guid.

Rezaei, F., Subramanian, S., Kalyanaraman, J., Lively, R.P., Kawajiri, Y., Realff, M.J., 2014. Modeling of rapid temperature swing adsorption using hollow fiber sorbents. Chem. Eng. Sci. 113, 62-76. doi:10.1016/j.ces.2014.04.002

Sato, S., Sato, K., Imamura, Y., 1975. Determination of the thermal shock resistance of graphite by arc discharge heating. Carbon N. Y. 13, 309-316. doi:10.1016/0008-6223(75)90035-4

Sharafat, S., Ghoniem, N., 2000. Summary of Thermo-Physical Properties of Sn , Comparison of Properties of Sn , Sn-Li , Li , and Pb-Li. UCLA-UCMEP-00-31 Rep. 1-51.

Siegel, N.P., Miller, J.E., Ermanoski, I., Diver, R.B., Stechel, E.B., 2013. Factors affecting the efficiency of solar driven metal oxide thermochemical cycles. Ind. Eng. Chem. Res. 52, 3276-3286.

Steinfeld, A., 2005. Solar thermochemical production of hydrogen-a review. Sol. Energy 78, 603-615. doi:10.1016/j.solener.2003.12.012

Steinfeld, A., Frei, A., Kuhn, P., Wuillemin, D., 1995. Solar thermal production of zinc and syngas via combined $\mathrm{ZnO}$-reduction and $\mathrm{CH}$ 4-reforming processes. Int. J. Hydrogen Energy 20, 793-804. 
Steinfeld, A., Sanders, S., Palumbo, R., 1999. Design aspects of solar thermochemical engineering - a case study: two-step water-splitting cycle using the $\mathrm{Fe} 3 \mathrm{O} 4 / \mathrm{FeO}$ redox system. Sol. Energy 65, 43-53.

Subasic, N., 1998. Thermodynamic evaluation of Sn-Zr phase diagram. Calphad 22, 157-165. doi:10.1016/S0364-5916(98)00021-2

Uher, C., 1991. 4.3.2 Temperature dependence of thermal conductivity of graphite, in: Madelung, O., White, G.K. (Eds.), Thermal Conductivity of Pure Metals and Alloys SE - 91, LandoltBörnstein - Group III Condensed Matter. Springer Berlin Heidelberg, pp. 430-439. doi:10.1007/10031435_91

Wei, B., Saadatfar, B., Fakhrai, R., Fransson, T., 2013. Review on the two-step-conversion of CO2 driven by solar energy. J. MacroTrends Energy Sustain. 1, 139-160.

Wetzel, T., Pacio, J., Marocco, L., Weisenburger, A., Heinzel, A., Schroer, C., Müller, G., Konys, J., Stieglitz, R., Fuchs, J., Knebel, J., Fazio, C., Daubner, M., Fellmoser, F., 2014. Liquid metal technology for concentrated solar power systems : Contributions by the German research program 2, 89-98. doi:10.3934/energy.2014.1.89

Xu, R., Wiesner, T.F., 2012. Dynamic model of a solar thermochemical water-splitting reactor with integrated energy collection and storage. Int. J. Hydrogen Energy 37, 2210-2223. doi:10.1016/j.ijhydene.2011.10.053

Zinkevich, M., Djurovic, D., Aldinger, F., 2006. Thermodynamic modelling of the ceriumoxygen system. Solid State Ionics 177, 989-1001. doi:10.1016/j.ssi.2006.02.044

\section{Figures}

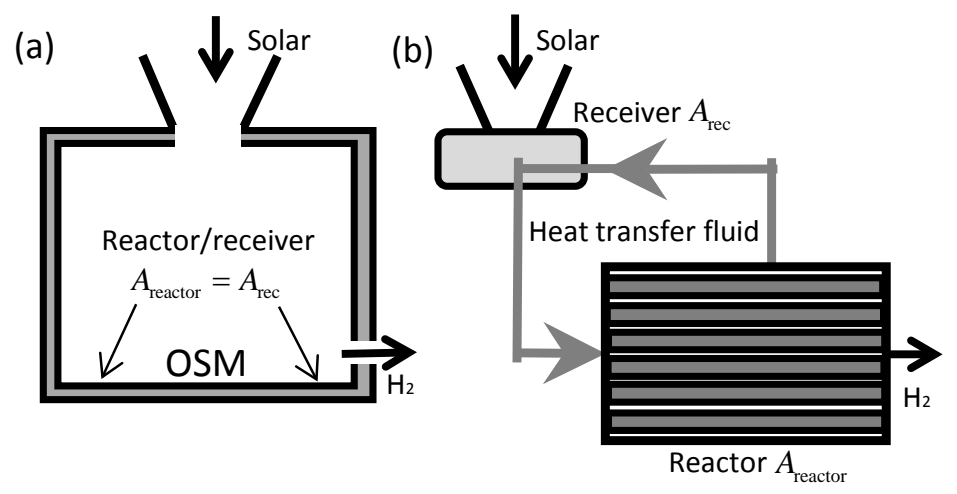

Fig. 1: Schematic of device to transfer solar energy to chemical energy 

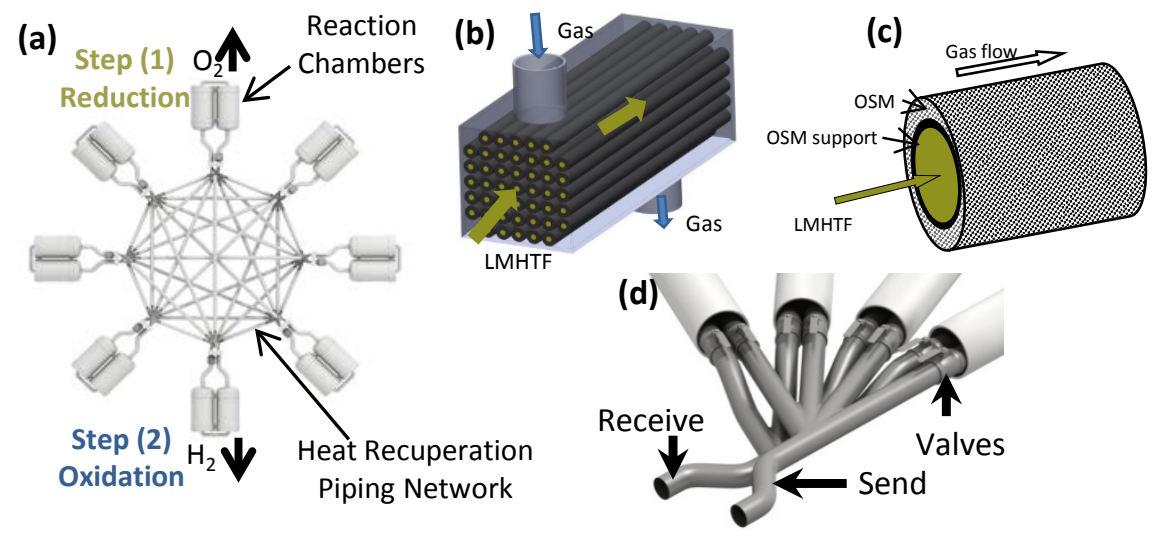

Fig. 2: (a) Thermochemical reactor schematic showing an array of sealed reaction chambers interconnected by a piping network. The chambers are denoted by two cylinder shapes and the piping network consists of pipes that do not intersect, but are overlaid on top of each other, so that each acts as a dedicated conduit for LMHTF circulation between a pair of chambers. At a given instant, two of the reaction chambers on opposite sides of the circle each undergo the reduction and oxidation steps respectively. (b) A schematic of an individual reaction chamber, which is consists of an array of pipes carrying LMHTF through the inner bore. (c) A cross-section of one tube inside of the chamber. (d) Each reaction chamber has a set of pipes that carry LMHTF to and from every other chamber in the circle.

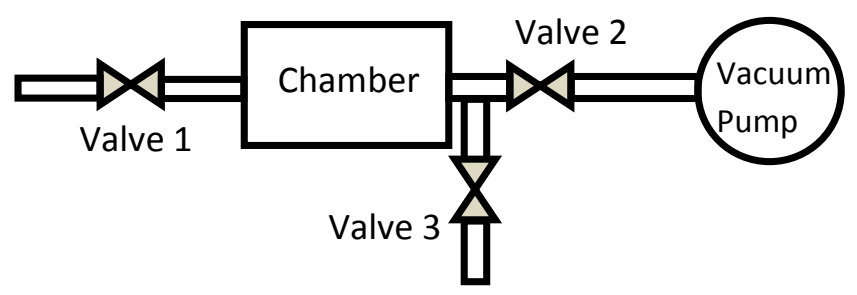

Fig. 3: Schematic of valve arrangement and sequence for controlling the gas environment 


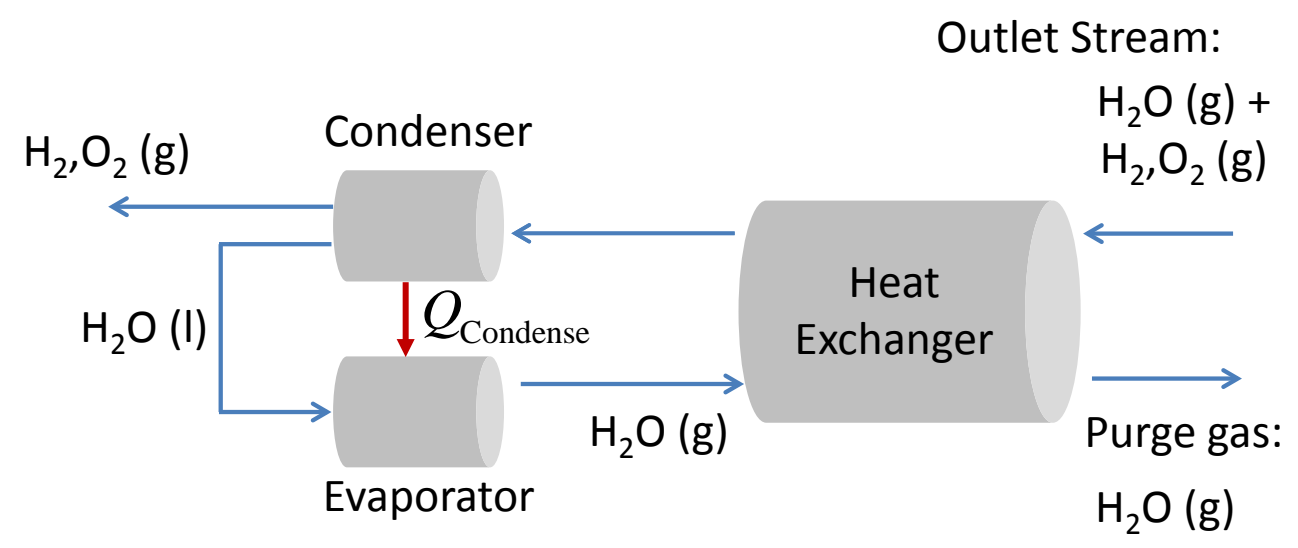

Fig. 4: A schematic of heat exchanger, condenser and evaporator Loop. The outlet stream from chamber with high temperature gas exchanges heat with the low temperature purge gas. Then the outlet stream enters a condenser to separate the liquid water, which will be supplied as purge gas after the evaporator. 

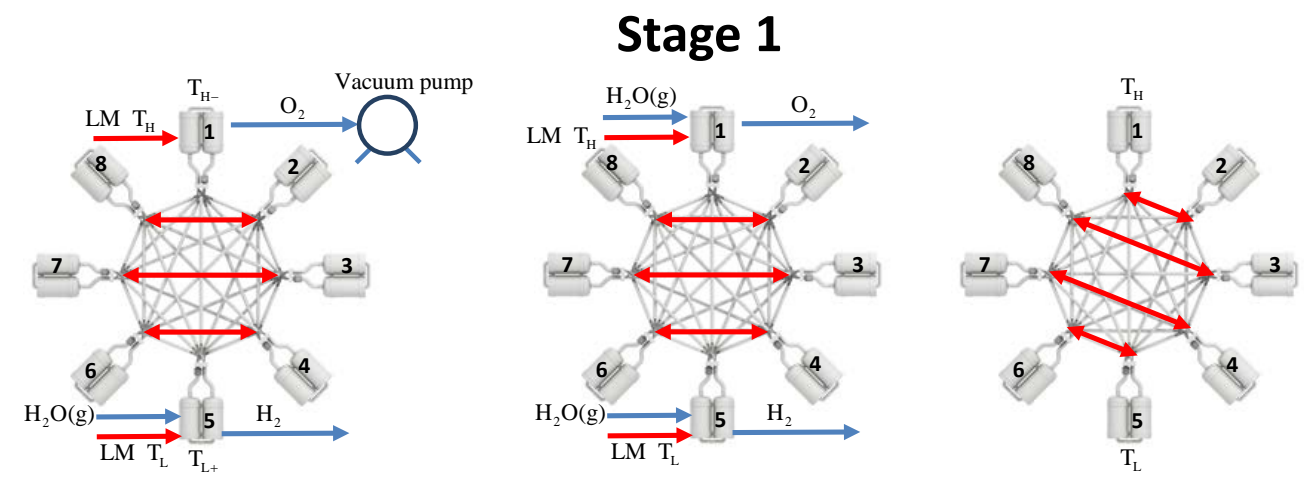

\section{Stage 2}
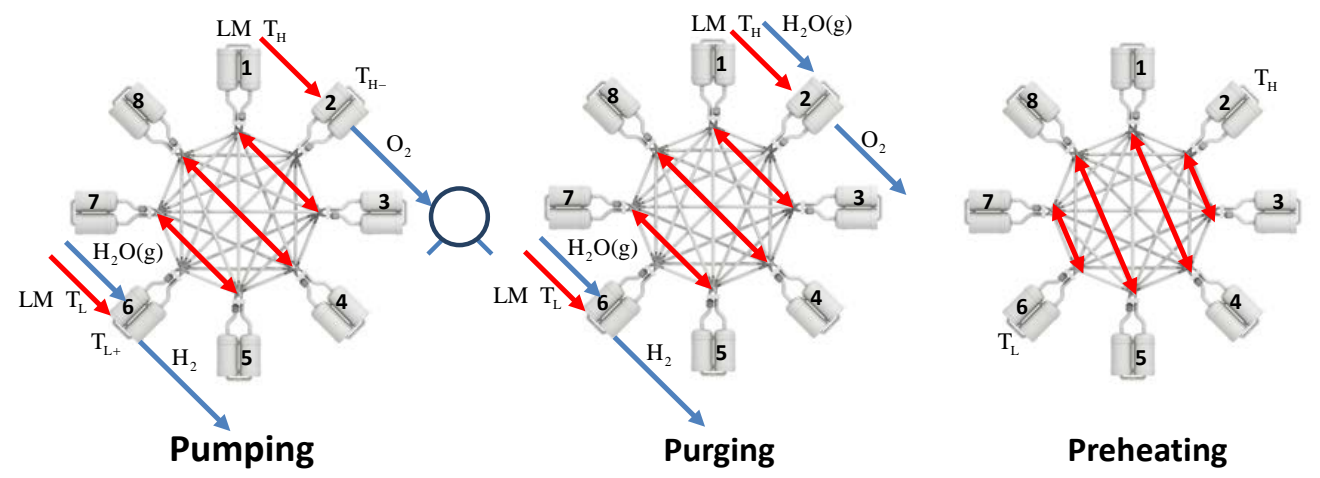

Fig. 5: A schematic diagram of the reaction and heat recuperation strategy for an eight-chamber reactor system. Red arrows denote which pipe channels are open and circulate the LMWF to exchange heat and bring different reaction chambers into equilibrium. Two grey cylinders represent the chambers and the numbers denote the chamber number. Only the first two stages are shown. The remaining six stages follow a similar sequence. Each stage includes three steps, pumping, purging and preheating.

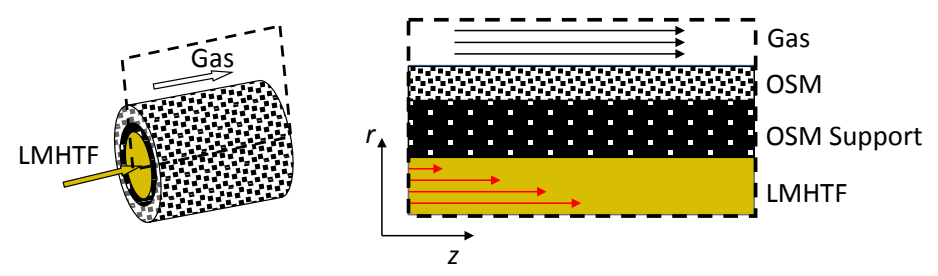

Fig. 6 Two-dimensional cross-sectional view of a single pipe 


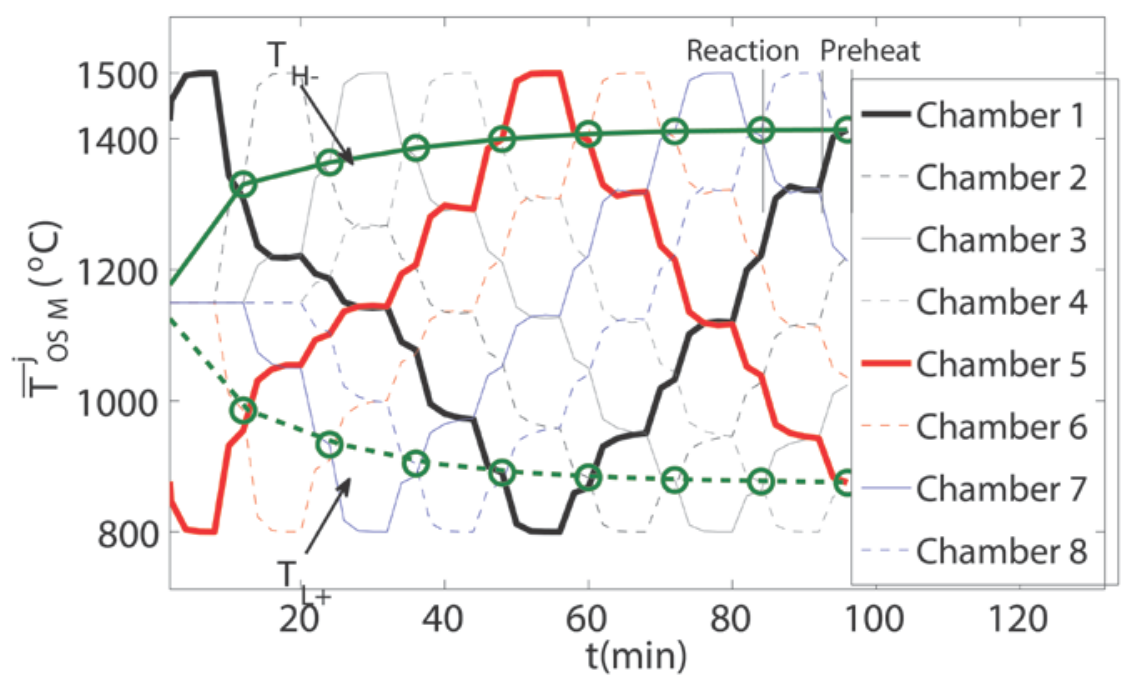

Fig. 7: Plot of the reaction chamber temperatures for one complete cycle, where each stage lasts 12 minutes.

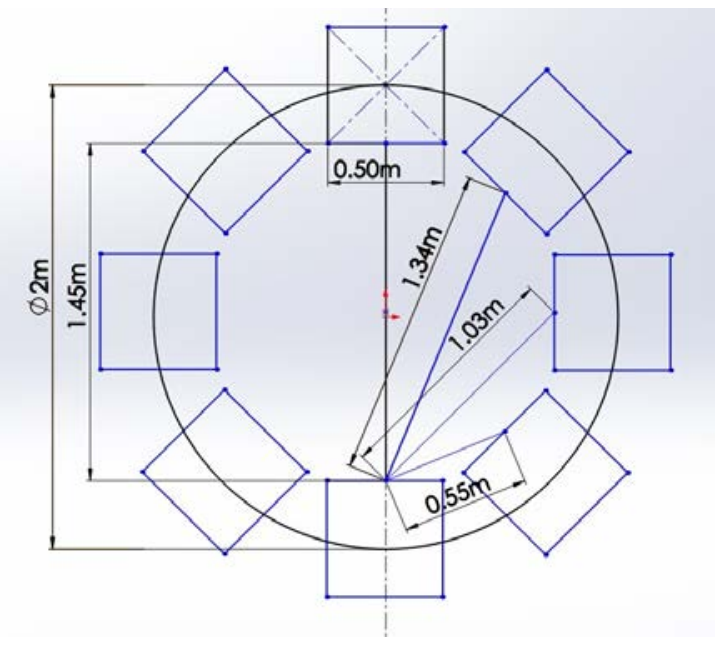

Fig. 8: A schematic diagram to compute the total pipe length of pipe network for eight chamber reactor. 


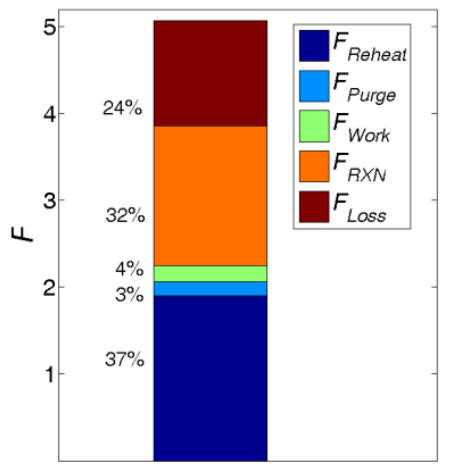

(a)

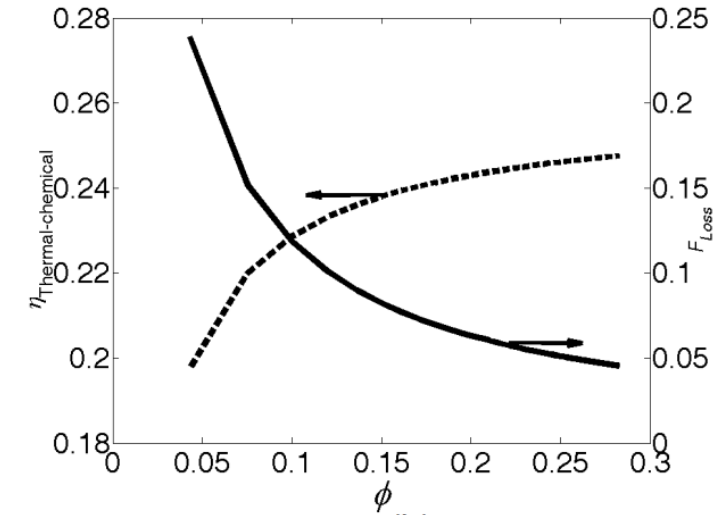

(b)

Fig. 9: (a) Examination of terms that limit the efficiency for the modelled eight chamber reactor system. (b) Plot of thermal chemical efficiency and heat loss fraction vs. volume to surface ratio. 